\title{
Non thermal techniques for dairy food processing applications
}

\author{
Shweta Saloni, Vipul Jaglan, Sindhu and Vaibhav Vyas
}

Received : $10.04 .2018 ;$ Accepted : 13.04 .2018

See end of the Paper for authors' affiliation

Correspondence to :

\section{Shweta Saloni} Department of Food Science and Technology, National Institute of Food Technology Entrepreneurship and Management, Kundli (Haryana) India
- Abstract : Processing and preservation of food is essential for value addition it require a variety of different methods and heat treatment is the most widely used methods due to its high efficiency on microbial inactivation. But exposure to high temperature causes undesirable changes such as reduction in organoleptic quality as well as reduction in heat-sensitive nutrients. Additionally, increasing consumer demand for minimally processed good quality and safe food products with natural flavor and taste, free from additives and preservatives, causes the need for the development of non-thermal methods for food preservation. There are a number of non-thermal methods used for food preservation all over the world. Foods can be non-thermally processed by High pressure processing, Pulsed electric field, Ohmic heating, Ultrasound treatment, etc. These novel technologies result in pathogen free foods with improved texture and improved shelf life. These techniques retain natural freshness, color and nutritive characteristics as compared to heat processing. The present review discusses the various novel non thermal techniques used today for food preservation.

- Key words : Non thermal, Processing, Non-thermal preservation, Temperature, Heating

- How to cite this paper : Saloni, Shweta, Jaglan, Vipul, Sindhu and Vyas, Vaibhav (2018). Non thermal techniques for dairy food processing applications. Internat. J. Agric. Engg., 11(Sp. Issue) : 142-148, DOI: 10.15740/HAS/IJAE/11.Sp. Issue/142-148. 